\title{
Primeras cirujanas de Chile
}

\author{
Pamela Pedrero B. ${ }^{1}$, Karen Rojas R. ${ }^{2}$, Lorena Pérez S. ${ }^{3}$, Claudia Albornoz G. ${ }^{4}$, \\ Jamile Camacho N. ${ }^{5}$, Katya Carrillo G. ${ }^{6}$, Macarena Fernández A. ${ }^{7}$, \\ Verónica Fonseca S. ${ }^{8}$, Ingrid Plass D. ${ }^{9}$, Carolina Rodríguez P. ${ }^{10}$ y Victoria Vargas V. ${ }^{11}$
}

'Servicio de Cirugía Instituto Nacional del Cáncer. Santiago

Servicio de Cirugi de Coquimbo. Coquimbo Chile.

3Servicio de Cirugía Hospital Regional de Talca. Talca, Chile. ${ }^{4}$ Servicio de Cirugía Hospita Clínico Universidad de Chile. Santiago, Chile.

Servicio de Cirugía Clínica Alemana. Santiago, Chile. ${ }^{6}$ Servicio de Cirugía Hospital Dra. Eloísa Díaz de la Florida. Santiago, Chile.

Servicio de Cirugía Hospita San Borja Arriarán. Santiago, Chile.

Servicio de Cirugía Hospita

Dr. Hernán Henríquez

Aravena. Temuco, Chile.

${ }^{9}$ Servicio de Cirugía Instituto

Nacional del Cáncer, Chile.

${ }^{10}$ Servicio de Cirugía Hospita de Curicó. Curicó, Chile.

'Servicio de Cirugía Hospita

Clínico Magallanes. Punta Arenas, Chile.

Recibido el 2020-12-01 y aceptado para publicación e 2021-01-04

Recibido año-mes-día y aceptado año-mes-día

Correspondencia a:

Dra. Pamela Pedrero B. ppedrerob1@yahoo.com

\section{First female surgeons of Chile}

In 2020, Chilean women surgeons gathered to discuss different problems affecting the development of a career in surgery. Through the history of the medicine and surgery in Chile, there have been several legal and cultural barriers that have kept women out of this specialty. One of this problem is the lack of knowledge of who were the first female surgeons and the lack of awareness of that there are women that can be leaders as well as male surgeons. So, generations of medical students and residents continue thinking that surgery might be a man's job. The purpose of this investigation is to reveal who went and are the first women surgeon in this country, not only in their time but also in their specialty, so we all know that there were and there still are pioneers among us.

Key words: humans; female; Chile; surgeons; physicians; women.

\section{Resumen}

En el año 2020 las cirujanas chilenas se reunieron en torno a la necesidad de trabajar para solucionar varios problemas que aquejan a la labor quirúrgica de la mujer en nuestro país, desde la formación de pregrado en adelante. Algunos de estos problemas son la poca visibilidad de la mujer en cirugía y la falta de reconocimiento de las cirujanas líderes y referentes del pasado y actuales. El objetivo de esta investigación fue indagar quienes habían sido las primeras cirujanas de Chile. La búsqueda fue difícil, ya que la información en la internet es escasa, por lo que se recurrió a entrevistas a discípulos y a algunas de las primeras cirujanas tanto en su especialidad o región del país. La cronología muestra lo reciente que ha sido la llegada de cirujanas a algunas regiones o la escasa representatividad de cirujanas en algunas subespecialidades. Esto se puede entender por barreras legales y culturales que han tenido que enfrentar las mujeres desde el ingreso a la carrera de medicina hasta el poder desarrollar la especialidad en cirugía a lo largo de la historia de este país.

Palabras clave: cirujanas; mujeres; médicos; Chile.

El año 2020 ha sido muy importante para las cirujanas de nuestro país, ya que a principios de marzo muchas cirujanas de distintos puntos del país, coincidimos en la necesidad de analizar la falta de visibilidad de la labor de las mujeres en cirugía. También nos dimos cuenta de que la idea de que somos muy pocas, no era precisa. Nos agrupamos inicialmente gracias a una aplicación telefónica y se obtuvieron varios testimonios con respecto a cómo ha sido y cómo es ser cirujana en la actualidad. Se presentó la necesidad de ahondar en temas como la poca representatividad de la mujer en la cirugía en nuestro país. ¿Sería acaso algún fenómeno local? ¿Sería sólo una sensación de las cirujanas? ¿Habría comunicaciones científicas al respecto? Se constituyó un grupo de trabajo de cirujanas de varias regiones de Chile en representación de todas. La finalidad era hacer un catastro de las cirujanas y becadas de cirugía general acreditadas en el presente. Luego, conocer su distribución por especialidad y tratar de dilucidar por qué, si la matrícula de estudiantes de medicina es equitativa en cuanto al género de las personas que ingresan cada año, el porcentaje de mujeres que optan a la especialidad de cirugía general de adulto sigue siendo baja. Se organizaron varios seminarios online para tratar temas relacionados con la falta de equidad para la mujer en el ambiente laboral, en periodo de formación médica y 
relacionados con la mujer y la cirugía. No obstante, surgieron otras dudas ¿por qué han estado invisibles las cirujanas en el pasado? ¿Quiénes son nuestras referentes? ¿Hay cirujanas en todas las subespecialidades? ¿Quiénes han sido las primeras cirujanas de nuestro país?

Históricamente la mujer chilena ha tenido dificultades para poder acceder a estudios superiores. La primera escuela de medicina de Chile se fundó en $1933^{1}$ y recién en 1877 se les permitió a las mujeres el ingreso a la universidad por un decreto firmado por Luis Amunátegui en el gobierno de Aníbal Pinto $^{2}$. En cuanto a la medicina, Eloísa Díaz fue la primera mujer egresada de esa carrera en Chile y América del Sur obteniendo su título el año $1887^{3,4}$. Sin embargo, hay que destacar que se siguió con la restricción a las mujeres ya que, hasta el fin de la década de los años 60 en el siglo XX, solo se disponía de un porcentaje reducido de la matrícula de las carreras de medicina para ellas, en las distintas universidades que la impartían ${ }^{5}$. Transcurriría más de un siglo desde que se fundó la primera escuela de medicina hasta que la mujer tuviese igualdad de acceso a estudiar esta carrera (Figura 1).

Un aspecto importante para tener interés en desarrollar una especialidad dentro de la medicina es conocer a los referentes de esta. En nuestra formación de pregrado crecimos viendo múltiples retratos de importantes maestros de la cirugía que adornan las paredes de cada facultad y/o servicio de cirugía, estudiando orgullosas (os) en impresionantes auditorios que ostentan en su entrada el nombre de algún destacado cirujano. Todos esos homenajes, por cierto, están merecidos y los atesoramos con orgullo. Pero el objetivo de esta investigación es el rescatar a las mujeres que han sido pioneras en la cirugía, que también son merecedoras de reconocimiento por sus méritos, entrega y trabajo impulsado por la pasión que nosotras también tenemos por esta bella especialidad. Además, se quiere destacar, no solo a la que fue la primera mujer en obtener un grado universitario de la especialidad quirúrgica en Chile, sino que el de dar a conocer los nombres y en breve, la trayectoria de aquellas cirujanas que nos han abierto el camino para poder desarrollarnos.

\section{Eleanira González Donoso, la primera de todas}

La Dra. Eleanira González Donoso fue la primera cirujana de Chile 6 . Nació el 17 de septiembre de 1892 en Santiago ${ }^{7}$. Titulada de médica el 4 de septiembre de 1925 en la Universidad de Chile ${ }^{8}$. Llega en 1926 a la clínica del Dr. Lucas Sierra donde comenzó su formación quirúrgica ${ }^{9}$. Siendo director general de Sanidad, el Dr. Sierra le encomendó, además, en 1927, la tarea de formar la Escuela de

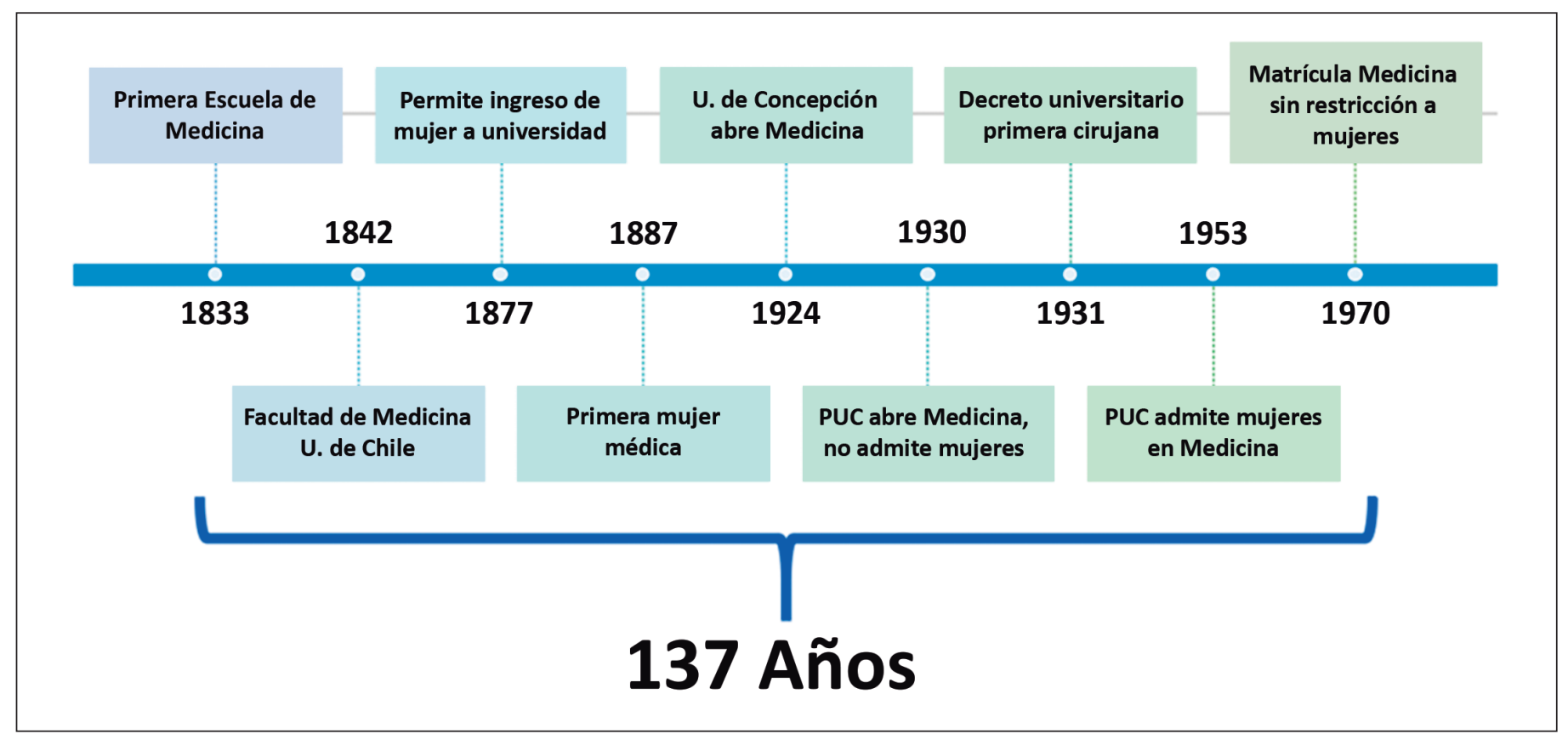

Figura 1. Línea de tiempo desde su fundación de primera escuela de medicina hasta el fin de las matrículas restringidas para el ingreso de mujeres a medicina. PUC: Pontificia Universidad Católica. U de Chile: Universidad de Chile. U de Concepción: Universidad de Concepción. 
Enfermeras Sanitarias. Esta tarea la concretaría junto a la Dra. Cora Mayers y a la enfermera estadounidense Sara Adams. Esta nueva escuela sería una de las bases de la escuela de enfermería universitaria de la cual la Dra. González sería distinguida directora, reconocida a nivel latinoamericano como autoridad universitaria $^{10-12}$.

En 1931 obtiene por decreto universitario el título de ayudante $1^{\circ}$ de la clínica quirúrgica de la Escuela de Medicina de la Universidad de Chile, única universidad en impartir medicina en esa época ${ }^{13}$. Además, el mismo año también por decreto se le confieren horas docentes de "higiene y puericultura" en el Instituto Pedagógico.

Fue ayudante de cátedra de cirugía cuando era una de las especialidades más competitivas de la carrera. Descrita por uno de sus colegas como la "estricta, siempre puntual y colaboradora del doctor Lucas Sierra", fue designada por él mismo como jefa de clínica, a pesar de los prejuicios y las protestas de varios cirujanos ${ }^{14,15}$.

Sus principales intereses fueron el bocio, cáncer de mama y uterino ${ }^{16}$. En una revista de la Liga Chilena contra el Cáncer de 1957, se reporta, que ella realizaba atenciones en el consultorio para el Diagnóstico Temprano del Cáncer de la Agrupación Médica Femenina en el Hospital San Borja, destacando que ese año se atendieron más de 500 mujeres con patología cervicouterina ${ }^{17}$. También consta su registro en la guía médica de Chile de $1959^{18}$. Fallece en 1972 a los 79 años ${ }^{19}$.

No se encontraron más datos de Eleanira González en las publicaciones existentes, pero es importante hacer notar el panorama con respecto a los derechos de la mujer de esa época, ya que la Dra.
González tuvo que abrirse paso y ganarse el respeto de sus colegas y pacientes en una sociedad en la cual las mujeres aún eran declaradas incapaces de tomar decisiones como, por ejemplo, elegir a sus gobernantes. En nuestro país, recién se les concedería el derecho a voto universal en $1949^{20}$ (Figura 2).

En contraste, los cirujanos ya se habían organizado mucho antes, con la fundación de la Sociedad de Cirugía de Chile en 1922, la cual era una elite de máximo 30 prominentes cirujanos. Por otro lado, los cirujanos de hospital, llamados los "cirujanillos" por la elite, fundarían su propia sociedad en 1931. Ambas se fundirían en una sola naciendo la actual Sociedad de Cirujanos de Chile ${ }^{21}$.

Según un artículo publicado en febrero de 2020, nuestra sociedad de cirujanos tiene un total de 1.240 socios, de los cuales sólo 141 son mujeres ${ }^{22}$.

Desde el grupo de trabajo de Cirujanas de Chile surge la idea, entonces, de encontrar a nuestras propias pioneras y referentes. La búsqueda fue muy difícil, no solo por el confinamiento de la pandemia que impidió acudir a investigar en archivos que no están digitalizados, sino porque las publicaciones al respecto son escasísimas y no ahondan en quienes fueron, quienes son, ni su trayectoria. El siguiente paso fue acudir a los registros de la Sociedad de Cirujanos y se encontró que desde 1949 a 2020 se ha elegido a 1 mujer presidenta, el 2010 (Dra. Jamile Camacho), una sola mujer elegida Maestra de la Cirugía en el año 2000 (Dra. Yvonne Pabst), solo 3 mujeres nombradas socias honorarias (Dra. Rose Marie Mege, Dra. Jamile Camacho y Dra. Verónica Silva) y ninguna cirujana ha sido nombrada socia emérita.

Teniendo en cuenta estos escasos datos, la infor-

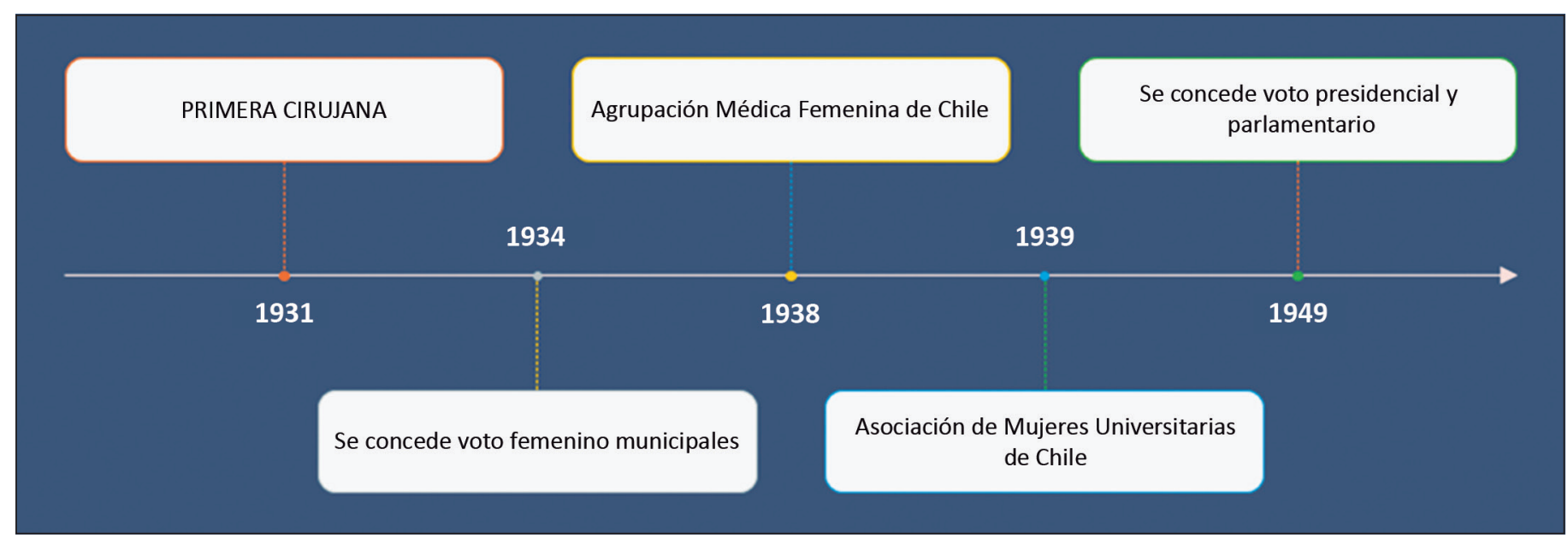

Figura 2. Línea de tiempo que correlaciona la obtención de título de la primera cirujana, movimientos femeninos e hitos cívicos en la primera mitad del siglo XX en Chile. 
mación con respecto a algunas de las pioneras de la cirugía en Chile se obtuvo mediante entrevistas a ellas misma o en algunos casos a sus discípulos. Continuaremos, entonces, esta revisión con nuestra maestra y con nuestra única presidenta hasta el momento, para proseguir con otras cirujanas quienes han sido las primeras en sus regiones y/o en sus especialidades (Figura 3).

\section{Dra. Ivonne Pabst Feller (1928-2014)}

Egresada de la Universidad de Chile en 1954, llega al Hospital San Juan de Dios, en un comienzo dedicándose a la cirugía general y cirugía de tiroides hasta que el profesor Alesandrini le encomienda la misión de encargarse del cáncer de mamas por ser una patología abandonada en ese tiempo. Por supuesto, la Dra. Pabst superó con creces la tarea. Fue jefa del equipo de patología mamaria de ese hospital, impulsora del desarrollo de nuevas técnicas como la punción quística diagnóstica y el ganglio centinela, además de encargarse personalmente de la quimioterapia de sus pacientes. Fue la creadora del equipo multidisciplinario de tratamiento oncológico del cáncer de mama y fue la presidenta fundadora de la Sociedad de Mastología. Recibió varios premios y reconocimientos en su larga carrera. Fue docente de numerosas generaciones de alumnos de pre y posgrado. De su escuela salieron destacadas cirujanas como las doctoras Soledad Torres y María Eugenia Bravo. Publicó libros. Declarada Maestra de la cirugía chilena el año 2000, no solo por su destreza quirúrgica, sino por su espíritu incansable, tenaz, humilde y generoso con sus conocimientos. Maestra y pionera por excelencia.

\section{Dra. Jamile Camacho Neira}

Egresada de la universidad de Concepción en 1983. Hizo residencia de cirugía general impartida por la Universidad de Chile en 1984 en Hospital Salvador. Fue una de las primeras becadas y cirujanas en ese centro. Realizó 15 años de cirugía general, para luego dedicarse de lleno a la patología mamaria. Ha superado barreras, ha sido docente, ha organizado diferentes unidades de patología mamaria en distintos centros públicos y privados. Ha participado en la directiva de la fundación "Yo Mujer". Ha hecho una notable carrera en la sociedad de cirujanos y llegó a ser la primera presidenta en el año 2010. Durante su mandato se reglamentaron los nuevos estatutos de la sociedad, lo que facilitó incorporar más socios y socias. Además, impulsó la creación del Departamento de Mamas de la Sociedad de Cirujanos y la difusión de los cursos de patología mamaria en regiones. Este año fue parte activa del grupo de trabajo de las Cirujanas de Chile $\mathrm{y}$, actualmente, del Departamento de Cirujanas. Sin duda, es una líder y nos sigue mostrando el camino.

\section{Dra. María Ibaceta Lizana}

Egresada de médico en el año 1966 de Concepción (examen de grado y título otorgado en ese

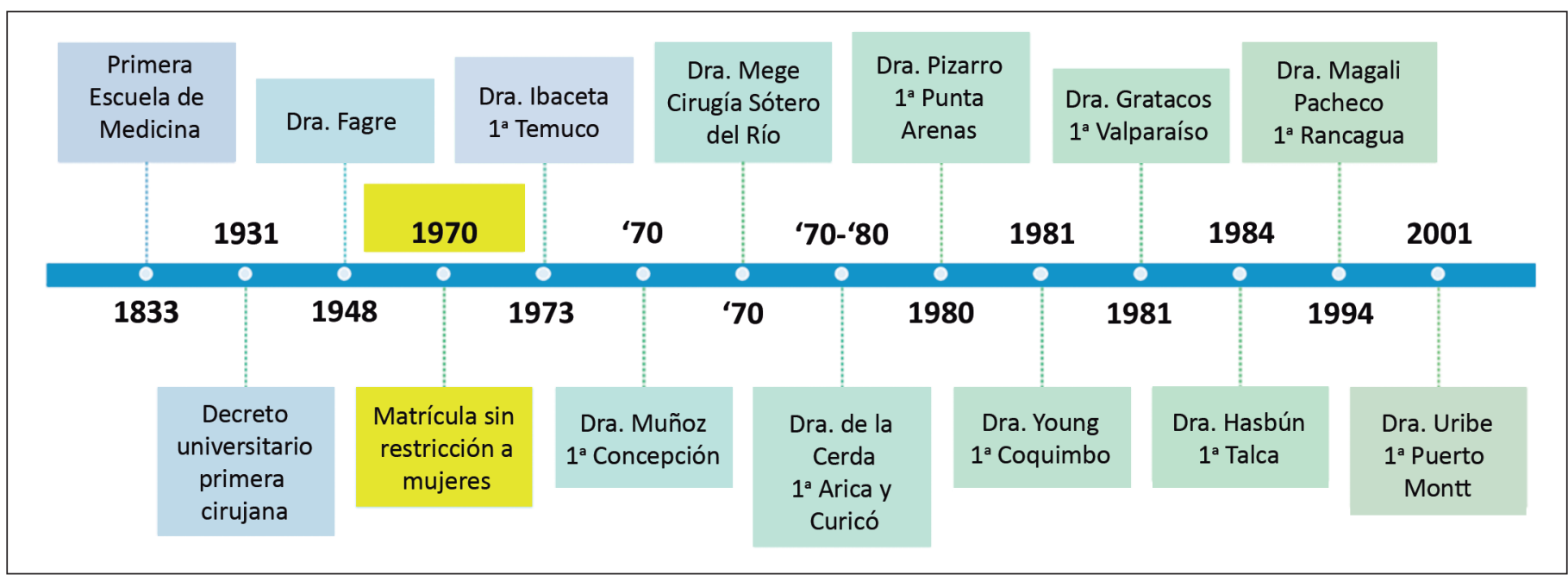

Figura 3. Línea de tiempo que muestra la aparición de las primeras cirujanas a lo largo de Chile. 
tiempo por la Universidad de Chile). Solo aceptaban $10 \%$ de mujeres en medicina. Fue médico general de zona y, posteriormente, hizo formación en cirugía general y cirugía plástica en el Hospital Salvador y en el Servicio de Quemados de HUAP entre 1970 y 1973 . Se trasladó a Temuco haciéndose cargo de desarrollar la especialidad en el hospital regional. Realizó varias estadías de capacitación con el renombrado Dr. Ivo Pitanguy en Brasil. Realizó 30 años de docencia en la Universidad de la Frontera. Fue jefa de pabellón, introdujo la informática en ese servicio, fue directora del hospital. Fue premiada al cumplir 50 años de médico en 2015, habiendo estado 43 como cirujana en el Hospital de Temuco.

\section{Dra. Rose Marie Mege Rivas}

Inició su formación de pregrado en la Pontificia Universidad Católica de Chile, se recibió en 1972. Hizo la formación quirúrgica en el Hospital Sótero del Río. Fue una de las formadoras de la escuela de cirugía de ese hospital desde sus orígenes en los años 70 . Miembro del equipo de cirugía digestiva y hepatobiliar. Impulsora de nuevas técnicas quirúrgicas, se capacitó en Tokio en ecografía desde principios de los años 80 , desarrollando la ecografía intraoperatoria y logrando importantes avances en la cirugía hepática. Además, formó un innovador departamento de ecografía quirúrgica exclusiva. Ha participado de numerosas publicaciones. Es una de las primeras cirujanas digestivas y miembro honorario de la Sociedad de Cirujanos de Chile.

\section{Dra. Zaira Pizarro Zoro}

Egresada en 1973 de la Universidad de Chile. Fue médico general de zona. Posteriormente, hizo residencia de cirugía en el Hospital Paula Jara Quemada. Se trasladó a Punta Arenas en 1980 y se convierte en la primera cirujana de esa ciudad. Allí formó la Unidad de Cirugía Maxilofacial y de Gran Quemado. En 1990 se traslada a Concepción donde en el Hospital Regional se hizo cargo de la patología mamaria creando la unidad propiamente tal en el año 1993. Propulsó la reconstrucción mamaria inmediata poscirugía oncológica. Hizo estadías de perfeccionamiento en Italia, España y Argentina. Además, fue docente de la Universidad de Concepción y también se hizo cargo de la docencia de pregrado y posgrado de patología mamaria además de residentes de cirugía.

\section{Dra. María Antonieta Muñoz Ujevich}

Egresada de Universidad de Concepción. Se abrió camino y fue la primera cirujana de esa ciudad. Se fue a España a hacer la subespecialidad de cirugía hepato biliar en el año 1982. Fue de las primeras en su región en realizar colecistectomías laparoscópicas. Fue la primera mujer de Sudamérica en ser miembro del American College of Surgeons. Fue jefa de servicio de cirugía del Hospital Regional de Concepción, jefa del departamento de cirugía de la Universidad de Concepción y secretaria académica de la facultad de medicina de la misma casa de estudios. Lamentablemente nos dejó tempranamente a los 57 años. Por su trayectoria y compromiso con la docencia la Universidad de Concepción le rindió homenaje en 2 oportunidades nombrando a la remodelada biblioteca de la facultad de medicina con su nombre y creando el Premio "Dra. María Antonieta Muñoz Ujevich" el cual se otorga anualmente a la mujer académica de la facultad que cumpla varios requisitos dentro de los que destacan la empatía, preocupación, cercanía y compromiso con los alumnos. Sin duda dejó huella en todos los que la conocieron.

\section{Dra. Teresa de la Cerda Alarcón}

Comenzó su carrera en 1973 en el Hospital Juan Noé de Arica y luego en Curicó. Fue la primera cirujana en ambas ciudades. En muchos proyectos se ha embarcado y terminado, incluso la formación de la unidad de cuidados críticos en esos años en $\mathrm{Cu}$ ricó. En el hospital del Salvador hizo su formación en cirugía plástica, donde también habían trabajado otras pioneras como las doctoras Ardiman y Fagre. Durante su carrera fue la gestora de la actual Sociedad de Cirugía Plástica y Reparadora de Chile, de la cual fue 2 veces su presidenta. Ha representado a Chile en la Federación Iberoamericana de Cirugía Plástica, ha sido presidenta en el Congreso Mundial de Cirugía Plástica (IPRAS) y actualmente es chairman del grupo de mujeres de ISAPS (International Society of Plastic Surgery).

\section{Dra. Bibiana Gratacos Naranjo}

Egresó de la Universidad de Chile en 1977. Hizo su residencia en la Universidad de Valparaíso en 1981. Fue la primera cirujana del Hospital Carlos Van Buren y del Hospital Dr. Eduardo Pereira, llegando a ser jefa de turno. En 1987 viaja a Suecia 
para formarse en cirugía vascular en el Instituto Karolinska y en el Hospital San Goran donde tuvo un cargo de planta en esa especialidad. Retorna a Chile y trabaja en Hospital Naval, luego en el Hospital de Quillota donde forma la Unidad de Accesos Vasculares para Hemodiálisis. Actualmente, se desempeña en el Hospital Carlos Van Buren donde mantiene cargo de artículo 44 y también realiza los accesos vasculares de ese centro.

\section{Dra. Alicia Young Valenzuela}

Comenzó la residencia de cirugía en el Hospital San Borja Arriarán en 1978. Fue la primera mujer residente en rotar por el Hospital del Tórax. Emigra a la cuarta región, donde fue la primera cirujana en el Hospital San Pablo de Coquimbo hasta 1986. Allí se adosó al equipo plástica y al de fisurados. Luego vuelve a Santiago al Hospital San Borja dedicándose a cirugía plástica hasta 2016 cuando se retira. Tuvo como mentora en el Hospital San Borja a la Dra. Rosa Fagre.

\section{Dra. Ana María Pacheco Frez}

Hizo su pregrado en Concepción donde conoció a la primera cirujana de ese centro y le quedaron grabadas las dificultades que algunos colegas le ponían para poder operar. Comenzó la residencia de cirugía en 1984. Fue la 4ª mujer residente en el Hospital Paula Jara Quemada. Ha hecho una larga y exitosa carrera en la cirugía de urgencia. Ha sido jefa de turno, jefa de Servicio de Asistencia Pública. Ha hecho varias estadías de capacitación en cirugía de trauma en el extranjero. Fue presidenta de la Sociedad Panamericana de Trauma (primera mujer de América del Sur en estar en este cargo). Recibió el Premio de la Sociedad Chilena de Medicina Crítica y de Urgencia por su aporte a la medicina intensiva $\mathrm{y}$ de urgencia en Chile. Fue distinguida por sus 30 años de servicio en HUAP. Ha sido miembro del directorio de la Sociedad de Cirujanos y del capítulo chileno del American College of Surgeons. Pionera en trauma $\mathrm{y}$, sin duda, una referente.

\section{Dra. Sandra Hasbún Atala}

Se tituló de médica en el año 1983 de la Universidad de Concepción. Comenzó su formación quirúrgica en 1984. Trabajó en Punta Arenas y luego en Talca. Fue la primera cirujana que tuvo el Hospital Regional de esa ciudad. Allí, junto a su maestro el
Dr. Roberto Tapia, desarrolló la especialidad de cirugía vascular periférica y fue de las primeras cirujanas en acreditar esta especialidad en Chile. Posteriormente, al ser designada como jefa de equipo, continuó con la incorporación de nuevas técnicas de cirugía endovascular y promovió la llegada de nuevos miembros para fortalecer su equipo.

\section{Dra. Magali Pacheco Méndez}

Egresó de la Universidad de Chile en 1986. Hizo residencia de cirugía en el Hospital Paula Jara Quemada, terminando en 1989. Llegó a Rancagua en 1994 siendo la primera cirujana del Hospital Regional. Se formó como vascular periférico y es miembro fundador de la Sociedad de Flebolinfología. Ha tenido una destacada carrera asistencial y gremial. Ha sido presidenta del capítulo regional de COLMED en 3 periodos, presidenta de la Sociedad de Cirujanos de la sexta región, vicepresidenta del Colegio Médico Nacional. Presidenta de FALMED tocándole dirigir con éxito una comisión negociadora, obteniendo beneficios para todos los médicos.

\section{Dra. Lorena Pérez Sotomayor}

Egresada de la Universidad de Chile. Hizo beca de cirugía en 1995 en el Hospital Salvador. Se traslada a la ciudad de Talca integrando el equipo de Tórax. Posteriormente, el año 2002, comienza la formación en oncología torácica de la Universidad de Chile. Es la primera cirujana de tórax con formación universitaria. Ha sido presidenta del capítulo del Maule de la Sociedad de Cirujanos. Coordinadora del curso de Cirugía de IV año de Medicina de la Universidad Católica del Maule desde el año 2006 al 2015. Jefa del Departamento de Cirugía desde el año 2009 a la fecha y es directora de Posgrado de Especialidades Médicas de la Facultad de Medicina de esta universidad desde el año 2017. También es la actual jefa del equipo de Cirugía Torácica del Hospital Regional de Talca.

\section{Dra. María Elena Molina Pezoa}

Hizo su pregrado en la Universidad Austral egresando en 1995. Fue médico general de zona. Posteriormente, hizo residencia de cirugía general en la Universidad Católica titulándose el año 2002. Continuó sus estudios con la subespecialidad de cirugía digestiva terminando el 2004. Fue la primera mujer en la historia de la Universidad Católica en 


\begin{tabular}{|l|l|l|}
\hline Claudia Albornoz G. & Cirugía Plástica & Región Metropolitana \\
\hline Jamile Camacho N. & Cirugía de Mamas & Región Metropolitana \\
\hline Katya Carillo G. & Coloproctología & Región Metropolitana \\
\hline Macarena Fernández A. & Coloproctología & Región Metropolitana \\
\hline Verónica Fonseca S. & Cirugía deTórax & Araucanía \\
\hline Ingrid Plass D. & Cirugía Cabeza y Cuello & Región Metropolitana \\
\hline Pamela Pedrero B. & Cirugía Digestiva Oncológica & Región Metropolitana \\
\hline Lorena Pérez S. & Cirugía deTórax & Maule \\
\hline Carolina Rodríguez P. & Cirugía Cabeza y Cuello & Maule \\
\hline Karen Rojas R. & Cirugía Bariátrica & Coquimbo \\
\hline Victoria Vargas V. & Cirugía General & Magallanes \\
\hline
\end{tabular}

Figura 4. En orden alfabético, grupo de trabajo representando a las cirujanas de Chile.

obtener este posgrado. Posteriormente, cursó estudios en cirugía coloproctológica y de piso pelviano en St. Mark's Hospital, Inglaterra, en el Hospital Clínico Universitario de Valencia y en el Hospital Juan Ramón Jiménez de Huelva. Es miembro titular de la Sociedad Chilena de Coloproctología desde el año 2011 y es la actual vicepresidenta de esa misma agrupación.

\section{Dra. Vivian Uribe Paredes}

Egresada de la Universidad Austral en 1997. Realizó la residencia de cirugía allí mismo siendo la primera cirujana egresada de esa universidad el año 2001. Comenzó su carrera en Ancud, trasladándose a Puerto Montt siendo la primera cirujana del Hospital Base. Fue jefa de turno varios años y directora del hospital. Formó la primera unidad de patología mamaria en 2006 siendo la única cirujana. Ha realizado un MBA y la subespecialidad de salud pública. Fue directora del Servicio de Salud de Reloncaví, subdirectora de gestión y, actualmente, es jefa de Servicio de Cirugía de su hospital y del equipo de mamas el cual cuenta con 6 miembros gracias a su gestión.

\section{Dra. Alejandra Gallego Cifuentes}

Realizó el pregrado en la Universidad de Santiago. Hizo el posgrado de cirugía general y la subespecialidad de cabeza y cuello de 2007 al 2009 en la Universidad de Chile. Miembro titular de sociedad de cirujanos, miembro del capítulo chileno del American College of Surgeons, Secretaria General del directorio de la Sociedad Chilena de Cabeza y Cuello y miembro del Comité de posgrado de la Universidad de Chile en la especialidad de Cabeza y Cuello. Es profesor asistente de la Universidad de Chile. Trabaja en el Hospital Barros Luco, en el Hospital Militar y en el Hospital Luis Calvo
Mackenna, haciendo cirugía oncológica de cabeza y cuello en niños. Ella señaló ser la segunda en obtener la subespecialidad de cabeza y cuello, sin embargo, la primera cirujana, la Dra. María Elena Rodríguez Rebolledo, falleció tempranamente a poco de obtener su grado.

\section{Dra. Rosa Fagre Fagre}

La Dra. Rosa Fagre Fagre, descendiente de libaneses, nacida en 1923, falleció en septiembre de 2009. Titulada en 1948, fue cirujana en el viejo Hospital San Borja de la Alameda, donde ahora están las torres San Borja. Se formó en cirugía plástica con el Dr. Rafael Urzúa Casas Cordero. Descrita por sus discípulos como una mujer maravillosa, extremadamente amable y humilde en su trato. Fue despedida a los 86 años, con un sentido homenaje por la Sociedad de Cirugía Plástica. Su historia ha sido rescatada por sus colegas y alumnos, ya que no hay información en la internet.

Todas quienes participamos en el grupo de trabajo de Cirujanas Chile, fuimos responsables de encausar en forma positiva toda la energía liberada tras el acercamiento de las cirujanas (Figura 4). Por primera vez, se discutían temas de equidad de género y ciclo vital en cirugía. Además, se llevó a cabo el proyecto de conformar el primer Departamento de Cirujanas de la SOCICH el cual fue aprobado el 7 de agosto de 2020. Lo logrado este año ha sido posible por la colaboración de casi 300 cirujanas y becadas de cirugía. Por esto, se puede considerar que todas quienes nos manifestamos de una u otra forma aportando ideas para mejorar la actividad y la labor de las cirujanas somos pioneras de la cirugía en Chile.

\section{Agradecimientos}

Dra. Cecilia Sepúlveda C.

Dr. Hernán Sudy P.

Dr. Hugo Rojas G.

Dr. César Paredes M.

\section{Responsabilidades éticas}

Protección de personas y animales. Los autores declaran que para esta investigación no se han realizado experimentos en seres humanos ni en animales.

Confidencialidad de los datos. Los autores declaran que en este artículo no aparecen datos de pacientes.

Conflictos de interés: no hay. 


\section{Bibliografía}

1. Laval E. Historia de las grandes etapas de la Cirugía en Chile (Lección inaugural del Curso de Cirugía de la Cátedra del Profesor Álvaro Covarrubias, 1956). Anales chilenos de la historia de la medicina 2006;16:243-52.

2. Decreto S/N 04-MAR-1877. Ministerio de Justicia, Culto e Instrucción Pública, Biblioteca del Congreso Nacional de Chile. https://www.archivonacional.gob. cl/616/w3-article-8046.html?noredirect=1 .

3. Biblioteca Nacional de Chile. "Eloísa Díaz”, en: Eloísa Díaz y Amanda Labarca. Memoria Chilena. Disponible en http:// www.memoriachilena.gob.cl/602/w3article-92520.html.

4. Eloísa Díaz Insunza: El derecho de la mujer a la educación universitaria. Revista de Educación 2016;375:2. Disponible en www.revistadeeducacion.cl

5. Sepúlveda C. Las mujeres en la medicina chilena: Desde los tiempos de Eloísa Díaz a la actualidad. Rev Med Chile 2019;147:1184-9.

6. Klimpel F. La mujer chilena (el aporte femenino al Progreso de Chile) 1910-1960. Editorial Andrés Bello; 1962. p. 158-60.

7. Chile, Registro Civil, 1885-1932, database with images, FamilySearch https://familysearch.org/ ark:/61903/1:1:VXPD-LYV: 10 April 2020), Eleanira González Donoso, Birth Registration; citing p. 23, certificate \#69, Registro Civil Archivo General (Civil Registry General Archives), Santiago; FamilySearch digital folder 004595021.

8. Índice de títulos conferidos por la Universidad de Chile en 1925. Anales de la Universidad de Chile, Pág. 917-962. doi: 10.5354/0717-8883.2013.27290 https://sye.uchile.cl/index.php/ANUC/ article/view/27290.

9. González I. El Juan Gandulfo de mis recuerdos. Anales Chil Hist Med. 1962;4:144-57.

10. Universidades de Latinoamérica. Vol. 5, no. 23 (noviembre 1954). http://132.247.171.154/handle/RepUDUAL/500.

11. Tobar I, Godoy M. Aporte al estudio comparativo de escuelas de enfermería, pág. 137 , primer congreso panamericano de enfermería, diciembre 1942, centenario de la Universidad de Chile, documento digitalizado) BIBLIOTECA NACIONAL DE CHILE. Enfermeras profesionalización y cuidado sanitario (1906-1950). Memoria Chilena. http:// www.memoriachilena.gob.cl/602/w3article-100622.html.

12. Núñez E, Jana A. El legado de las enfermeras sanitarias en el cuidado de la salud chilena. Ciencia y enfermería [Internet]. 2017 [citado el 15 de octubre de 2020];23:113-24. Disponible en: https://dx.doi.org/10.4067/S071795532017000300113.

13. Actas de las sesiones del consejo universitario. Anales de la Universidad de Chile, pp. 255-393.doi: 10.5354/07178883.2019.53899 https://anales.uchile.cl/ index.php/ANUC/article/view/53899.

14. Chateau G. Lucas Sierra. Anales Chil Hist Med. 1966;8:53-73.

15. Guzmán L. El espíritu del Profesor Lucas Sierra. Anales Chil Hist Med. 1966;8:2040.

16. Cornejo G. Contribución del Profesor Sierra al desarrollo de la cirugía y la enseñanza de la medicina en Chile. Anales Chil Hist Med. 1966;8:41-52.

17. Decimoctava Memoria Anual: 19561957. Santiago de Chile: Liga Chilena Contra el Cáncer, 1957. https:// www.bcn.cl/obtienearchivo?id=doc umentos/10221.1/55548/1/203154. pdf\&origen=BDigital.

18. Guía médica nacional: profesiones médicas y paramédicas. Santiago de Chile: S.N.S., 1959. https://obtienearchivo. bcn.cl/obtienearchivo?id=document os/10221.1/55354/2/188355.pdf.

19. Chile, registros de cementerios, 1821-2015, Database with images, FamilySearch https://familysearch.org/ ark:/61903/1:1:Q2HY-5WX6: 10 April 2020), Eleanira González Donoso, 11 Jan 1972; citing Death, Santiago, Región Metropolitana de Santiago, Chile, Cementerio General de Santiago (General Cemetery of Santiago), Chile.

20. Biblioteca Nacional de Chile. "Voto femenino", en: Elecciones, sufragio y democracia en Chile (1810-2012) Memoria Chilena. http://www. memoriachilena.gob.cl/602/w3article-93508.html.

21. Cubillos L. Historia de la Sociedad de Cirujanos de Chile: Primera parte. Rev Chil Cir. 2012;64:3128. https://scielo.conicyt.cl/scielo. php?script $=$ sci_arttext\&pid=S0718 40262012000300017\&lng=es. http://dx.doi.org/10.4067/S071840262012000300017.

22. Valenzuela M. La mujer cirujano: ¿Todavía un "cuento para niñas rebeldes"? Rev Cirugía. 2020;72:7-8. https://dx.doi.org/10.35687/s245245492020001322. 


\section{Conceptos divergentes de recurrencia en hernia inguinal}

\section{Divergent concepts in inguinal hernia recurrence}

\section{Estimado Dr. Yarmuch:}

Actualmente existe divergencia, entre los distintos cirujanos, en el concepto e interpretación de recurrencia en hernia inguinal, lo que obliga mediante una secuencia lógica de argumentos e ideas, a analizar algunas afirmaciones. Han transcurrido 16 años desde la publicación de Cisneros $\mathrm{HA}^{1}$ y no se ha logrado unificar criterios.

Son varios los sinónimos empleados para la hernia inguinal recurrente: recidivante, repetida, iterada, reiterada, secundaria, reproducida o replicada ${ }^{1}$.

La palabra recurrir deriva del latín (recurrere) que significa volver una cosa al sitio o lugar original de donde salió. En medicina significa: reaparecer una enfermedad después de intermisiones. Recurrente: que vuelve a ocurrir o a aparecer, especialmente después de un intervalo de desaparición temporal ${ }^{2}$. Según el concepto etimológico, para que una hernia se considere como recurrente deberá reaparecer en el mismo sitio anatómico específico operado previamente.

El grupo HerniaSurge ${ }^{3}$ no declara, en las directrices o guías internacionales para el tratamiento de las hernias de la región inguinal, un concepto o definición de hernia inguinal recurrente; sin embargo, este mismo grupo declara que, actualmente, las técnicas abiertas que usan malla (principalmente la de Lichtenstein como estándar de oro) son las más usadas para la reparación de la hernia inguinal primaria (directa, indirecta, mixta), al mismo tiempo que permiten reforzar de forma preventiva otra zona vulnerable mediante el material protésico ${ }^{4}$.

Más del $70 \%$ de las intervenciones quirúrgicas primarias se realizan por una hernia inguinal indirecta y más del $60 \%$ de los defectos herniarios inguinales recurrentes aparecen en la pared posterior (hernia inguinal directa) $)^{5}$.

La clasificación sobre la hernia inguinal primaria y recurrente, que declara la Sociedad de Hernia Europea y describen Klinge U. et al. ${ }^{6}$, se ajusta más al concepto de recurrencia que aparece en el mismo sitio anatómico específico operado previamente.

La clasificación propuesta por Campanelli, citada por Cuccurullo D et al. ${ }^{7}$ para hernias ingui- nales recurrentes, a partir de la segunda recidiva, no delimita sitio anatómico específico operado previamente y la clasifica como multirrecurrente; por esta razón, la segunda recurrencia se debe a la reaparición del defecto en la misma región inguinal operada.

Para el tratamiento quirúrgico actual de la hernia inguinal recurrente, un grupo de cirujanos no tiene en cuenta el tipo específico de la hernia primaria (directa, indirecta, mixta), por lo tanto, el manejo será siempre con la misma técnica ya que el objetivo es reparar y reforzar las zonas vulnerables; mientras que otros cirujanos individualizan la técnica de reparación, de acuerdo con el tipo específico de hernia que presenta cada paciente ${ }^{8}$.

La hernia inguinal recurrente se define como la reaparición de un defecto herniario en el mismo sitio anatómico específico de la hernia primariamente operada después de una desaparición quirúrgica temporal; de otro modo, se tratará de una segunda hernia primaria y diferente. Si se ha aplicado una técnica de reparación libre de tensión con reforzamiento preventivo de otras zonas vulnerables mediante material protésico y reaparece otro defecto en un sitio diferente al primariamente operado, se denominará reparación inguinal profiláctica fallida con defecto residual en la región inguinal ${ }^{9}$.

Sin embargo, y en términos prácticos, se habla de recidiva de hernia inguinal cuando aparece de nuevo un defecto herniario en la misma región inguinal operada -independientemente del tipo de hernia original-, lo cual quiere decir que si el paciente presentaba un defecto indirecto y ahora el saco parece provenir del piso y se considera como hernia directa, representa una recidiva, pues el objetivo al hacer la primera cirugía era ocluir el defecto herniario y reforzar los potenciales sitios de aparición de otras hernias ${ }^{10}$

La tasa de recurrencia es el indicador más frecuente con el que se miden los resultados en la reparación de la hernia inguinal. Esta disparidad de criterios trae consigo confusiones en los índices reales de recurrencia publicados por los diferentes autores, donde algunos obtendrán mayores índices que otros al utilizar una técnica específica si manejan la recurrencia desde el punto de vista de la región inguinal o desde el punto de vista anatómico específico (directa, indirecta). Sería prudente unificar criterios y establecer o validar un concepto único para evitar diferencias en los índices de recurrencia de cada técnica quirúrgica.

Fernando Karel Fonseca Sosa 


\section{Bibliografía}

1. Cisneros HA. Recurrencia en hernia inguinal. ¿Qué significa? ¿Es un problema semántico? ¿Es problema de lenguaje e interpretación? ¿Es el error del milenio? Cir General 2004;26:260-4.

2. Diccionario Real Academia Española de la Lengua. Disponible en: https://dle.rae.es/recurrente? $\mathrm{m}=$ form.

3. The Hernia Surge Group. International guidelines for groin hernia management. Hernia 2018;22:1-165.

4. Cisneros HA. ¿Logrará algún procedimiento en las próximas décadas desplazar a la hernioplastia inguinal de Lichtenstein como el estándar de oro? Rev Hispanoam Hernia 2020;8:53-5.

5. Gutiérrez Chambi JC. Factores relacionados a la recidiva de hernias inguinales en pacientes hospitalizados en cirugía del hospital III Goyeneche. [tesis]. Perú: Universidad Nacional de San Agustín; 2018.

6. Klinge U, Pross M. Repair Principles and Materials.
En: Schumpelick V, Arlt G, Conze J, editores. Hernia Surgery. 5th ed. Germany: Thieme; 2019. pp. 47.

7. Cuccurullo D, Reggio S. Classification of Inguinal and Abdominal Wall Hernia. En: Campanelli G, editor. The Art of Hernia Surgery. Switzerland: Springer; 2018. pp. 27-28.

8. Mayagoitia JC. Clasificación de las hernias inguinales. En: Mayagoitia JC, Cisneros HA, Ramírez LR, editores. Hernias de la pared abdominal. Tratamiento actual. México: Editorial Alfil; 2015.p. 151-155.

9. Fonseca Sosa FK, Lorenzo Mestril A, Rey Vallés YS, Llópiz Salazar R. Factores de riesgo asociados a la hernia inguinal recurrente en el adulto mayor. Rev Chil Cir. 2019;71:61-5.

10. Mayagoitia JC, Cisneros HA. Hernias inguinales recurrentes o recidivantes. En: Mayagoitia JC, Cisneros HA, Ramírez LR, editores. Hernias de la pared abdominal. Tratamiento actual. México: Editorial Alfil; 2015. p. 285. 